\title{
Escore de ambiente construído relacionado com a prática de atividade física no lazer: aplicação numa região de baixo nível socioeconômico
}

\section{Built environment score related to leisure-time physical activity: application in a region of low socioeconomic status}

\author{
Alex Antonio Florindo ${ }^{1,2}$ \\ Leandro Martin Totaro Garcia ${ }^{1,3}$ \\ Vanessa Valente Guimarães ${ }^{1,2}$ \\ Emanuel Péricles Salvador ${ }^{1,3}$ \\ Homero Fonseca Filho ${ }^{2,4}$ \\ Rodrigo Siqueira Reis ${ }^{5,6}$ \\ José Cazuza de Farias Junior 7,8
}

Resumo - São escassas as evidências sobre a relação entre atividade física de lazer (AFL) e ambiente construído no Brasil, especialmente em áreas de baixo nível socioeconômico. Sendo assim, objetivou-se descrever indicadores de ambiente construído relacionados à prática de AFL de adultos de uma região de baixo nível socioeconômico. Trata-se de estudo transversal com 767 adultos que viviam no Distrito de Ermelino Matarazzo, em São Paulo, em 2007. A variável dependente foi a prática de AFL (em minutos por semana). As independentes foram 35 variáveis ambientais avaliadas por auditagem em 30 setores censitários. A AFL foi correlacionada (coeficiente de Spearman) com todas as variáveis ambientais e aquelas que tiveram $\mathrm{p}<0,20$ foram selecionadas para compor dois escores (variáveis com $\mathrm{p}<0,05$ tiveram peso 2): Instalações (escore 1) e Segurança para o deslocamento (escore 2). Ambos os escores foram categorizados em quintis e o ambiente construído foi composto pela soma dos quintis destes escores, com variação de 2 a 10 pontos. Quartis de ambiente construído foram calculados e o primeiro quartil foi considerado como um ambiente mais adequado para AFL. O escore de ambiente construído teve 14 variáveis significativamente associadas com a AFL e consideradas como atributos importantes para esta prática no lazer.

Palavras-chave: Atividade física; Atividades de Lazer; Avaliação; Meio Ambiente; Saúde Pública.

Abstract - There is little evidence on the relationship between leisure-time physical activity (LTPA) and built environment in Brazil, especially in areas of low socioeconomic status. The objective of this study was to describe indicators of built environment score related to LTPA for adults living in a region of low socioeconomic status. We conducted a cross-sectional study of 767 adults living in Ermelino Matarazzo district in São Paulo, 2007. The dependent variable was the practice of LTPA (in minutes per week). Independent variables were 35 environment variables that were evaluated by an audit instrument in 30 census tracts. LTPA was correlated (Spearman coefficient) with all environment variables, and variables that had $p<0.20$ were selected to compose two scores (variables with $p<0.05$ had weight value of 2): Infrastructure (score 1) and Safety for commuting (score 2). Both scores were divided in quintiles and the built environment was composed by the sum of the quintiles of these scores, ranging from 2 to 10 points. Quartiles of built environment were calculated and the first was considered as the most adequate environment for LTPA. The built environment score had 14 variables significantly associated with LTPA and were considered as important attributes for this leisure practice.

Key words: Environment; Evaluation; Leisure activities; Physical activity; Public health.

\begin{abstract}
1 Universidade de São Paulo. Grupo de Estudos e Pesquisas Epidemiológicas em Atividade Física e Saúde. São Paulo. SP. Brasil.
\end{abstract}

2 Universidade de São Paulo. Escola de Artes. Ciências e Humanidades. São Paulo. SP. Brasil.

3 Universidade de São Paulo. Faculdade de Saúde Pública. São Paulo. SP. Brasil.

4 Universidade de São Paulo. Grupo de Estudos e Pesquisas em Infraestrutura de Dados Espaciais. São Paulo. SP. Brasil.

5 Pontifícia Universidade Católica do Paraná. Escola de Saúde e Biociências. Grupo de Pesquisas em Atividade Física e Qualidade de Vida. Curitiba, PR, Brasil.

6 Universidade Federal do Paraná Programa de Pós-Graduação em Educação Física. Curitiba, PR, Brasil.

7 Universidade Federal da Paraíba. Programa Associado de Pós-Graduação em Educação Física. João Pessoa, PB, Brasil.

8 Universidade Federal da Paraíba. Grupo de Estudos e Pesquisas em Epidemiologia da Atividade Física. João Pessoa, PB, Brasil.

Recebido em 30/05/12 Revisado em 11/07/12 Aprovado em 03/09/12

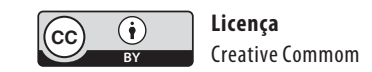




\section{INTRODUÇÃO}

A atividade física (AF) é um complexo comportamento humano e diversos são os aspectos que influenciam a adoção da sua prática, compreendendo variáveis individuais e ambientais ${ }^{1}$. Entre estes aspectos, o ambiente construído tem recebido uma crescente atenção por parte de pesquisadores. $\mathrm{O}$ ambiente construído relacionado à AF compreende o conjunto de atributos entorno das pessoas, incluindo os naturais (por exemplo, clima e topografia) e construídos (por exemplo, proximidade de locais para realizar AF, disponibilidade de calçadas e faixas de pedestres) ${ }^{2}$. As evidências disponíveis demonstram que características do ambiente como densidade de destinos, facilidade de acesso, disponibilidade de áreas verdes e espaços abertos para recreação, número de intersecções de ruas e a segurança (geral e no trânsito) estão associadas com a prática de $\mathrm{AF}$, principalmente em pessoas adultas e idosas ${ }^{3}$.

Alguns aspectos têm limitado a compreensão dos achados sobre ambiente construído e AF. Primeiramente, dada a diversidade de aspectos que compõem o ambiente construído, a sua avaliação é complexa e inclui entrevistas por questionários (percepções sobre o ambiente) e ainda métodos objetivos como auditagem de segmentos e sistemas de informações geográficas com o uso de imagens de satélites ${ }^{4}$. Além disto, o ambiente construído apresenta características contextuais inerentes às comunidades. Por exemplo, o desenho das cidades é notadamente menos conectado e denso na América do Norte do que em países da América do Sul e de colonização ibérica e portuguesa ${ }^{5}$. Finalmente, o conjunto atual de evidências é originado, em sua grande parte, de países de renda alta ${ }^{6}$.

Os estudos conduzidos no contexto brasileiro têm enfocado as percepções sobre o ambiente e sua relação com a $\mathrm{AF}^{7-11}$, porém ainda são escassas as evidências sobre a relação entre a AF e o ambiente construído, medido de maneira objetiva. Recentemente, um estudo com adultos do município de Curitiba, PR, mostrou que a quantidade de academias e a proximidade de centros de recreação das residências dos participantes estavam associadas com a prática de AF moderada no tempo de lazer ${ }^{12}$.

Na revisão de literatura não foram encontrados estudos similares no Brasil, mais especificamente em áreas de baixo nível socioeconômico, as quais podem apresentar características distintas das comunidades de renda mais alta. Em parte, isto acontece pela baixa disponibilidade de informações obtidas pelos sistemas de informações geográficas fornecidas por órgãos públicos brasileiros. Uma alternativa para esta limitação é o uso de métodos mistos que combinem auditagem do ambiente às informações de imagem fornecidas por satélites, que podem ser uma alternativa para comunidades menos desenvolvidas economicamente ${ }^{4}$. Portanto, o objetivo deste estudo foi descrever indicadores de a mbiente construído relacionados à prática de AF no tempo de lazer de adultos que vivem em uma região de baixo nível socioeconômico na zona leste do município de São Paulo, SP. 


\section{PROCEDIMENTOS METODOLÓGICOS}

O presente estudo foi desenvolvido como parte da pesquisa "Atividade física e sua relação com variáveis individuais e ambientes na população adulta do distrito de Ermelino Matarazzo da zona leste do Município de São Paulo". Tratou-se de um estudo transversal de base populacional, em que a coleta de dados foi realizada no ano de $2007^{9-10,13}$. O estudo foi aprovado pelo Comitê de Ética em Pesquisa da Faculdade de Saúde Pública da Universidade de São Paulo (protocolo número 1488/2006).

O distrito de Ermelino Matarazzo está localizado na zona leste do município de São Paulo. A zona leste é a região mais populosa do município e tem mais de quatro milhões de habitantes (aproximadamente $30 \%$ da população do município). O índice de desenvolvimento humano (IDH) médio dos 21 distritos que compõe as zonas leste 1 e 2, pela divisão geográfica do município de São Paulo, é de 0,790, abaixo do IDH do município de São Paulo (IDH=0,841). Ermelino Matarazzo está na $72^{\text {a }}$ posição no IDH de 96 Distritos do município de São Paulo e de acordo com dados de 2010 do Instituto Brasileiro de Geografia e Estatística, o distrito tem uma área de $8,95 \mathrm{~km}^{2}$ e 113.615 residentes, com uma densidade populacional de 15.419 habitantes por $\mathrm{km}^{2}$.

De uma amostra inicial de 890 sujeitos de 35 setores censitários do inquérito de base domiciliar, foram utilizados 767 sujeitos que residiam em 30 setores censitários, dado que houve a exclusão de cinco setores censitários por problemas na avaliação do ambiente. Todo o detalhamento da amostragem está descrito em Florindo et al. ${ }^{9}$.

A amostra para este estudo foi composta de $58 \%$ de mulheres, $56,3 \%$ com idade de 18 a 59 anos e 59,7\% com escolaridade de até sete anos completo e não completo.

\section{Avaliação da atividade física}

A AF no tempo de lazer foi avaliada por meio do questionário internacional de AF (IPAQ) versão longa (neste estudo foi usado somente o módulo de AF no lazer). Foram somados o tempo de prática em atividades de caminhada, em atividades moderadas e em atividades vigorosas ${ }^{14-15}$. O ponto de corte utilizado para a classificação da AF no tempo de lazer foi de 150 minutos ou mais por semana ${ }^{15-16}$.

\section{Avaliação do ambiente construído}

- Instrumento de auditagem de ruas

Para a avaliação das estruturas das ruas foi utilizado um instrumento elaborado especificamente para este estudo. Este instrumento seguiu algumas recomendações do International Environment and Physical Activity Network (IPEN) (<www.ipenproject.org $>$ ) e foi adaptado para a realidade do distrito de Ermelino Matarazzo. O formulário permite avaliar detalhadamente diversos itens estruturais das ruas como a quan- 
tidade de conexões, segurança para pedestres, cobertura de árvores, iluminação pública, qualidade e existência das calçadas e do transporte público, a estética das construções, além de conservação e poluição. Todas as avaliações foram feitas por setores censitários de acordo com a especificação do Instituto Brasileiro de Geografia e Estatística (Figura 1). A coleta de dados foi feita entre junho a dezembro de 2007 por quatro avaliadores que receberam 10 horas de treinamento. O processo de desenvolvimento e os indicadores de reprodutibilidade demonstraram que a maioria das variáveis categóricas teve concordância acima de $70 \%$ e a maior parte das variáveis quantitativas não apresentaram diferenças entre as médias. Mais detalhes podem ser obtidos em Bortoni et al. ${ }^{17}$.

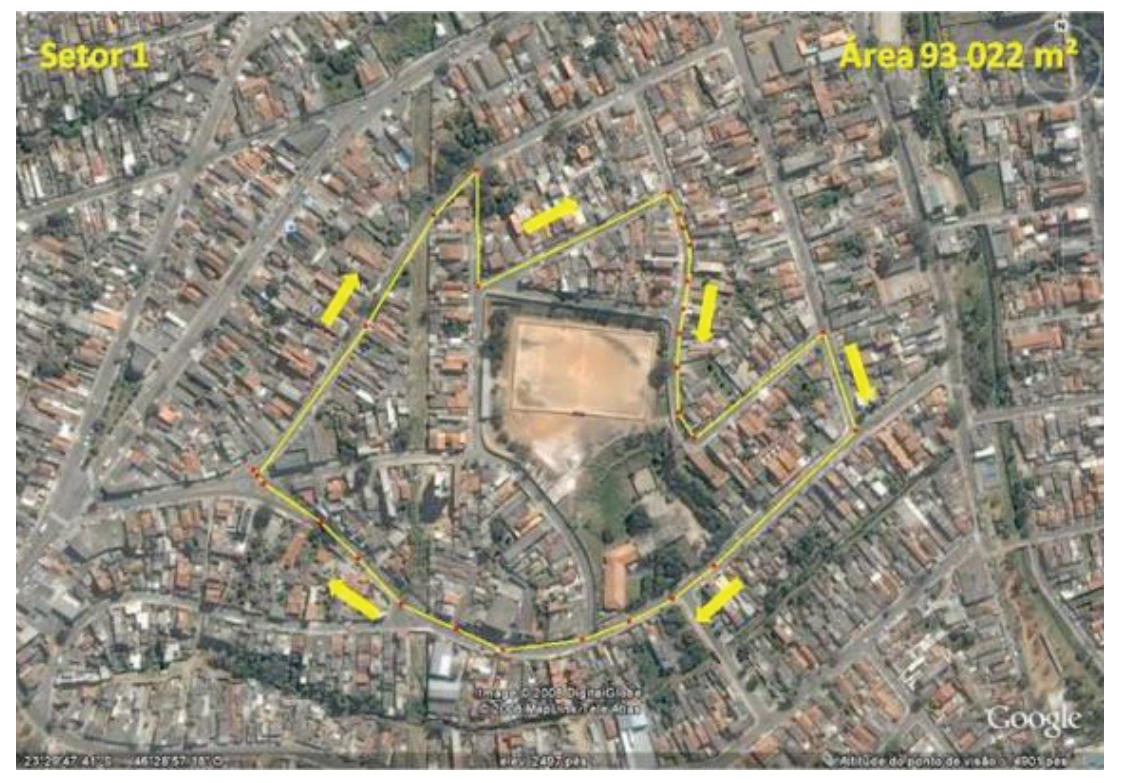

Figura 1. Imagem de um setor censitário avaliado.

- Dados secundários da Prefeitura de São Paulo sobre itens do ambiente construído

Foi realizada uma pesquisa na base de dados da prefeitura do município de São Paulo para localizar os itens estruturais de serviços públicos dentro do distrito de Ermelino Matarazzo como a quantidade de clubes, praças, parques e unidades básicas de saúde (<www.prefeitura.sp.gov.br/cidade/ secretarias/subprefeituras/ermelino_matarazzo >).

- Dados de sistema de posicionamento por satélite

Foi calculada a área total aproximada de cada setor censitário em metros quadrados $\left(\mathrm{m}^{2}\right)$. Para isso, os avaliadores percorreram o perímetro de cada setor censitário com receptores de sinais GNSS/GPS (Global Navigation Satellite System/Global Positioning System), modelo EtrexVista Cx da marca Garmim e coletaram dados de posição no terreno a partir dos sinais dos satélites (Figura 1). Em seguida, os dados foram processados no programa MapSource, versão 6.10.2, para calcular os valores aproximados de área. A média das áreas dos 30 setores censitários pesquisados em Ermelino 
Matarazzo foi de $72.876,5 \mathrm{~m}^{2}$ (desvios-padrão $=83.010,1 \mathrm{~m}^{2}$ e variação de $23.187,0 \mathrm{~m}^{2}$ a $\left.485.033,0 \mathrm{~m}^{2}\right)$.

\section{Análise dos resultados}

Os dados da avaliação do ambiente construído foram agrupados por setores censitários por meio da soma de cada variável avaliada dentro dos setores. Foram geradas 35 variáveis (Quadro 1) escolhidas com base nas recomendações do IPEN < www.ipenproject.org > e em estudos anteriores que analisaram o ambiente construído em outros países $^{2,18-19}$ e percepção do ambiente relacionada com a prática de $\mathrm{AF}$ em amostras de adultos e idosos de Ermelino Matarazzo ${ }^{9,13,20}$. As variáveis foram testadas em duas etapas:

- Análise de correlação de Spearman entre os minutos de AF no lazer das pessoas com cada variável ambiental avaliada de forma objetiva. Nesta fase, foram selecionadas para a criação do escore as variáveis que obtiveram $\mathrm{p}<0,20^{21}$;

- Elaboração do escore de ambiente construído. Nesta fase foram criados dois escores: a) escore de instalações relacionadas com a AF; b) escore de segurança para o deslocamento. Ambos os escores foram ajustados pela área total do setor censitário medida por GPS. Foi atribuído peso 2 para as variáveis que tiveram resultados de coeficiente de correlação de $\mathrm{p}<0,05$ na fase 1 de análise. A partir da criação destes dois escores, foram calculados os quintis de cada um e somados para a elaboração do escore final que variou de 2 até 10 pontos. Para finalizar, foram calculados os quartis do escore final e foi considerado como ambiente mais adequado para a prática de AF no lazer o quartil superior do escore.

Posteriormente, foi verificado se existiam diferenças no escore de ambiente construído segundo características sociodemográficas, utilizando-se o teste de Mann-Whitney e de Kruskal-Wallis. Finalmente, foi realizada análise de Mann-Whitney para verificar se existiam diferenças de médias entre os escores de ambiente construído e os níveis de AF no lazer dos residentes nos setores censitários. Todas as análises foram feitas no software SPSS 15.0.

\section{RESULTADOS}

Do total de 35 variáveis avaliadas inicialmente (Quadro 1), sete variáveis apresentaram $\mathrm{p}<0,05$ (quantidade de clubes públicos, quantidade de faixas de pedestres, quantidade de pistas de caminhada, quantidade de igrejas, quantidade de escolas, quantidade de escolas de esporte e quantidade de associações de moradores de bairro) e sete variáveis tiveram $p>0,05$ e $\mathrm{p}<0,20$ (quantidade de semáforos, quantidade de luminárias nos postes, quantidade de academias, quantidade de campos de futebol, quantidade de calçadas, quantidade de calçadas com largura maior do que um metro e quantidade de ruas com pontos com esgoto a céu aberto) (Tabela 1). Somente a variável quantidade de lombadas não foi considerada para as 
próximas etapas, pois apesar de tem um valor $\mathrm{de} p=0,156$, houve correlação inversa com a prática de AF no lazer.

Quadro 1. Variáveis do ambiente construído avaliadas em cada setor censitário do distrito de Ermelino Matarazzo, São Paulo, SP, 2007.

\begin{tabular}{|l|l|}
\hline Variáveis do ambiente construído & Quantidade de lombadas \\
\hline Quantidade de clubes públicos & Quantidade de semáforos \\
\hline Quantidade de praças & Quantidade de calçadas \\
\hline Quantidade de escolas & Quantidade de calçadas com largura maior que 1 metro \\
\hline Quantidade de academias & $\begin{array}{l}\text { Quantidade de obstáculos permanentes nas calçadas } \\
\text { (buracos, postes, árvores, desníveis) }\end{array}$ \\
\hline Quantidade de escolas de esporte & Quantidade de árvores \\
\hline Quantidade de campos de futebol & Quantidade de luminárias nos postes \\
\hline Quantidade de pistas de caminhada para AF & Quantidades de pontos de ônibus \\
\hline Quantidade de igrejas & $\begin{array}{l}\text { Quantidade de vielas (ruas que não permitem a circulação de } \\
\text { carros) }\end{array}$ \\
\hline $\begin{array}{l}\text { Quantidade de bares } \\
\text { Quantidade de unidades básicas de saúde }\end{array}$ & Quantidade de ruas sem saída \\
\hline Quantidade de associações de moradores de bairro & Quantidade de casas abandonadas \\
\hline Quantidade de estabelecimentos de comércios e serviços & Quantidade de carros \\
\hline Quantidade de domicílios & $\begin{array}{l}\text { Quantidade média de circulação de veículos em } 1 \text { minuto nas } \\
\text { ruas }\end{array}$ \\
\hline $\begin{array}{l}\text { Densidade populacional (total de pessoas/área do setor } \\
\text { censitário) }\end{array}$ & Quantidade de postos policiais \\
\hline $\begin{array}{l}\text { Densidade de comércios/serviços (total de comércios e servi- } \\
\text { ços/total de residências) }\end{array}$ & Quantidade de ruas com lixo (sem sinal de coleta) \\
\hline Quantidade de ruas planas & Quantidade de ruas com esgoto a céu aberto \\
\hline Quantidade de conexões de ruas & Quantidade de ruas com poluição do ar \\
\hline Quantidade de faixas de pedestres & \\
\hline
\end{tabular}

As variáveis que formam cada escore e os procedimentos para realizar os cálculos estão descritas a seguir:

- Escore de acesso a instalações: 1.1. Calculou-se o escore de acesso a instalações associadas à $\mathrm{AF}$ por meio da seguinte equação: [(n ${ }^{\circ}$ clubes $+\mathrm{n}^{\circ}$ pistas de caminhada $+\mathrm{n}^{\circ}$ igrejas $+\mathrm{n}^{\circ}$ escolas $+\mathrm{n}^{\circ}$ escolas de esportes $+\mathrm{n}^{\circ}$ associações de moradores de bairro) $\mathrm{x} 2+\left(\mathrm{n}^{\circ}\right.$ academias $+\mathrm{n}^{\circ}$ campos de futebol)] / tamanho do setor $\left(\mathrm{em} \mathrm{m}^{2}\right)$; 1.2. Dividiu-se os valores dos resultados do escore de acesso a instalações em quintis;

- Escore de segurança para o deslocamento: 2.1. Calculou-se o escore de segurança para o deslocamento por meio da seguinte equação: [ $\left(\mathrm{n}^{\circ}\right.$ faixas de pedestres $\times 2)+\left(n^{\circ}\right.$ semáforos $+n^{\circ}$ luminárias $+n^{\circ}$ calçadas $+\mathrm{n}^{\circ}$ calçadas com largura maior do que um metro) $-\mathrm{n}^{\circ}$ ruas com esgoto a céu aberto] / tamanho do setor $\left(\mathrm{em} \mathrm{m}^{2}\right) ; 2.2$. Dividiu-se os valores dos resultados do escore de segurança para o deslocamento em quintis; 
Tabela 1. Coeficientes de correlação de Spearman $\left(r_{\text {sho }}\right)$ entre minutos de AF no tempo de lazer e as variáveis de ambiente construído, distrito de Ermelino Matarazzo, São Paulo, SP, 2007.

\begin{tabular}{|c|c|}
\hline Variáveis do ambiente construído & $r_{\text {sho }}(p)$ \\
\hline Quantidade de clubes públicos & $0,08(0,037)^{*}$ \\
\hline Quantidade de praças & $0,01(0,720)$ \\
\hline Quantidade de escolas & $0,08(0,035)^{*}$ \\
\hline Quantidade de academias & $0,06(0,100)^{*}$ \\
\hline Quantidade de escolas de esporte & $0,12(0,001)^{*}$ \\
\hline Quantidade de campos de futebol & $0,06(0,087)^{*}$ \\
\hline Quantidade de pontos de caminhada para AF & $0,11(0,002)^{*}$ \\
\hline Quantidade de igrejas & $0,09(0,012)^{*}$ \\
\hline Quantidade de bares & $-0,04(0,278)$ \\
\hline Quantidade de unidades básicas de saúde & $0,02(0,540)$ \\
\hline Quantidade de associações de moradores de bairro & $0,08(0,025)^{*}$ \\
\hline Quantidade de estabelecimentos de comércios e serviços & $0,01(0,288)$ \\
\hline Quantidade de domicílios & $-0,02(0,659)$ \\
\hline Densidade populacional & $-0,01(0,699)$ \\
\hline Densidade de comércios/serviços & $0,03(0,407)$ \\
\hline Quantidade de ruas planas & $0,02(0,560)$ \\
\hline Quantidade de conexões de ruas & $0,03(0,346)$ \\
\hline Quantidade de faixas de pedestres & $0,08(0,024)^{*}$ \\
\hline Quantidade de lombadas & $-0,05(0,156)^{*}$ \\
\hline Quantidade de semáforos & $0,06(0,078)^{*}$ \\
\hline Quantidade de calçadas & $0,07(0,072)^{*}$ \\
\hline Quantidade de calçadas com largura maior que 1 metro & $0,06(0,105)^{*}$ \\
\hline Quantidade de obstáculos permanentes nas calçadas & $0,03(0,469)$ \\
\hline Quantidade de árvores & $0,03(0,425)$ \\
\hline Quantidade de luminárias nos postes & $0,08(0,064)^{*}$ \\
\hline Quantidades de pontos de ônibus & $-0,03(0,478)$ \\
\hline Quantidade de vielas & $-0,03(0,431)$ \\
\hline Quantidade de ruas sem saída & $-0,01(0,830)$ \\
\hline Quantidade de casas abandonadas & $-0,01(0,836)$ \\
\hline Quantidade de carros & $0,03(0,388)$ \\
\hline Quantidade média de circulação de veículos em 1 minuto nas ruas & $0,04(0,288)$ \\
\hline Quantidade de postos policiais & $0,01(0,999)$ \\
\hline Quantidade de ruas com lixo & $0,01(0,907)$ \\
\hline Quantidade de ruas com esgoto a céu aberto & $-0,06(0,106)^{*}$ \\
\hline Quantidade de ruas com poluição do ar & $-0,01(0,778)$ \\
\hline
\end{tabular}

* Variáveis com $\mathrm{p}<0,20$.

- Escore final de ambiente construído: 3.1. Somou-se os quintis do escore de acesso a instalações + quintis do escore de segurança para o deslocamento. Esta soma pode variar de 2 até 10; 3.2. Dividiu-se o escore de ambiente construído em quartis. O maior quartil é considerado como um ambiente mais adequado para a prática de $\mathrm{AF}$ no lazer.

A média do escore de ambiente construído foi de $6,0(\mathrm{dp}=2,1)$, com variação de 3,0 a até 10,0 . Nas análises descritivas, observou-se que $31,2 \%$ dos setores censitários foram classificados com um ambiente mais adequado 
para prática de AF no lazer (quartil superior). Não houve diferenças significativas do escore de ambiente construído segundo sexo, idade e níveis de escolaridade (Tabela 2).

Tabela 2. Descrição do escore ambiente construído segundo características sociodemográficas dos residentes em de Ermelino Matarazzo, São Paulo, SP, 2007.

\begin{tabular}{|lcccccc}
\hline Variáveis & $\mathrm{n}$ & Mínimo & Máximo & Média & $\mathrm{dp}$ & $\mathrm{p}$ \\
\hline Sexo & & & & & & \\
\hline Masculino & 322 & 3 & 10 & 6,01 & 2,06 & 0,388 \\
\hline Feminino & 445 & 3 & 10 & 5,94 & 2,06 & \\
\hline Escolaridade & & & & & & \\
\hline Até três anos & 198 & 3 & 10 & 5,92 & 1,90 & 0,722 \\
\hline De quatro a sete anos & 260 & 3 & 10 & 6,08 & 2,07 & \\
\hline De oito a 11 anos & 220 & 3 & 10 & 6,02 & 2,19 & \\
\hline 12 anos ou mais & 89 & 3 & 10 & 5,86 & 2,06 & \\
\hline Idade & & & & & & \\
\hline 18 a 39 anos & 260 & 3 & 10 & 6,11 & 2,12 & 0,398 \\
\hline 40 a 59 anos & 172 & 3 & 10 & 5,95 & 2,14 & \\
60 anos ou mais & 335 & 3 & 10 & 5,93 & 1,97 & \\
\hline
\end{tabular}

Nas comparações dos valores médios do nível de AF no lazer dos moradores dos setores censitários segundo tipo de ambiente para a prática (quartis superior e inferior), observou-se uma diferença significativa com maior média de AF para os residentes em ambientes classificados como mais adequados (Tabela 3 ).

Tabela 3. Valores de média e desvios-padrão (dp) dos minutos de AF segundo escore de ambiente construído e valores de média e dp do escore de ambiente construído segundo níveis de AF dos residentes de Ermelino Matarazzo, São Paulo, SP, 2007.

\begin{tabular}{|c|c|c|c|}
\hline \multirow[t]{2}{*}{ Variáveis } & \multicolumn{3}{|c|}{ Minutos de AF no lazer } \\
\hline & Média & $\mathrm{dp}$ & $\mathrm{p}$ \\
\hline Escore de ambiente construído & & & $<0,001$ \\
\hline Quartil superior & 102,9 & 207,8 & \\
\hline \multirow[t]{2}{*}{ Quartil inferior } & 69,2 & 177,4 & \\
\hline & \multicolumn{3}{|c|}{ Escore de ambiente construído } \\
\hline AF no lazer & & & 0,009 \\
\hline Atingem as recomendações* & 6,4 & 2,0 & \\
\hline Não atingem as recomendações* & 5,9 & 2,1 & \\
\hline
\end{tabular}

*Pelo menos 150 minutos por semana de prática de atividade física no tempo de lazer.

\section{DISCUSSÃO}

O presente estudo mostrou que as variáveis: clubes públicos, igrejas ou centros religiosos, escolas, escolas de esportes, associações de moradores de bairro, academias de exercícios físicos, campos de futebol, faixas de pedestres, semáforos, luminárias, calçadas, calçadas com largura maior do que um metro e ruas sem poluição/esgoto a céu aberto foram correla- 
cionadas com a prática de AF no lazer de adultos e compuseram o escore final de ambiente construído em Ermelino Matarazzo, zona leste de São Paulo, SP.

Métodos de avaliação objetiva do ambiente por meio de instrumentos de auditagem (observação e contagem de itens) ou por sistemas de informações geográficas (SIG) para avaliar a associação do ambiente com a prática de AF têm sido muito utilizados em países de renda alta $^{2-3}$. Estes países dispõem de bons bancos de dados espaciais de sua infraestrutura urbana. Os SIG e as infraestruturas de dados espaciais permitem a sobreposição, a análise espacial e a combinação de imagens de satélites de dados da infraestrutura urbana com dados observados por auditagem e com dados secundários. A maioria dos estudos tem utilizado indicadores como a densidade populacional, uso misto do solo, acesso as instalações de recreação, padrões das ruas, estado das calçadas, tráfego de veículos, indicadores de segurança, desenho de edifícios, trânsito de pessoas, topografia (declividade) das ruas, áreas verdes e índices compostos por todos os indicadores citados².

Das 35 variáveis ambientais avaliadas inicialmente, as 14 que foram selecionadas para compor o escore de ambiente construído estão dentro dos fatores relacionados aos modelos de ambiente que podem influenciar a prática de $\mathrm{AF}^{2,9,13,18-19}$. Foi observada maior média de minutos de prática de $\mathrm{AF}$ no tempo de lazer nas pessoas residentes em setores censitários com ambiente classificado como adequado de acordo com o escore de ambiente construído deste estudo. Além disso, a média do escore foi maior no grupo de pessoas que praticou 150 minutos ou mais por semana de AF no tempo de lazer.

No entanto, ainda existem poucos estudos avaliando o ambiente de forma objetiva e propondo indicadores para verificar a relação com a prática de AF em países como o Brasil. Tal característica limita as comparações dos achados deste estudo com a literatura. $\mathrm{O}$ único estudo encontrado foi realizado na cidade de Curitiba e teve como objetivo verificar a associação de itens do ambiente avaliado de forma objetiva com a prática de AF no tempo de lazer em adultos ${ }^{12}$. Os autores realizaram inquérito telefônico para avaliar a AF no tempo de lazer e levantaram indicadores objetivos do ambiente por meio de informações secundárias na prefeitura da cidade. Os dados foram inseridos em SIG para a análise do entorno e foi padronizado um raio de 500 metros em ao redor das residências onde foi avaliada a AF. As únicas variáveis associadas com a prática de AF foram obtidas com a densidade de academias e a renda dos setores censitários.

Estudo realizado na cidade de Bogotá no ano de 2005 verificou a associação do ambiente avaliado de forma objetiva com a prática de AF no lazer em adultos ${ }^{22}$. Foram selecionados 27 bairros levando em consideração o nível socioeconômico da região (baixo, médio e alto) e foi avaliado o nível de AF no tempo de lazer por meio do IPAQ versão longa em 1.315 adultos. Os resultados mostraram que a topografia (declividade) foi associada inversamente e a densidade de parques e proximidade de estações de transporte público foram associadas positivamente com a prática de AF no tempo de lazer. 
É importante ressaltar que ambos os estudos realizados em Curitiba e em Bogotá utilizaram exclusivamente dados compatíveis com SIG para avaliar o ambiente relacionado com a prática de AF. Apesar de estes estudos terem sido realizados em países de renda média (Brasil e Colômbia), a disponibilidade de dados compatíveis com SIG ainda é restrita em muitas regiões destes países e o uso de ferramentas como os instrumentos de auditagem de ruas são mais acessíveis e permitem um maior detalhamento de informações ${ }^{4}$.

Uma variável importante para a prática de AF no lazer no estudo com adultos de Curitiba foi a presença de academias de exercício físico próximas às residências ${ }^{12}$. A presença de academias dentro dos setores censitários também foi importante e entrou na composição do escore deste estudo. Estudos realizados na mesma região e com parte da amostra usada no presente estudo que analisaram o ambiente percebido mostraram também que a presença de clubes, campos de futebol e igrejas ou templos religiosos foram associados com a prática de AF no lazer em adultos e idosos ${ }^{9,13}$. As escolas de esporte e pistas de caminhada são estruturas específicas para a AF no tempo de lazer. As escolas são estruturas voltadas para o ensino, mas que em algumas regiões periféricas de São Paulo também podem servir para o lazer, principalmente porque podem ser abertas para a comunidade nos finais de semana por meio do programa Escola da Família do Governo do Estado de São Paulo. Neste programa, universitários são contratados para organizar atividades de lazer e cultura para as famílias em algumas escolas que permanecem abertas sábados e domingos. No ano de 2012, existiam no município de São Paulo 389 escolas estaduais com este programa implantado $<$ http://escoladafamilia.fde.sp.gov.br $>$.

As associações comunitárias têm importante papel no desenvolvimento local, transformando de forma consciente a realidade e o ambiente - inclusive social - do seu entorno ${ }^{23}$. Estas associações podem aumentar o capital social da comunidade em que estão inseridas, ou seja, podem fortalecer os vínculos e as interações sociais entre os moradores e aumentar os benefícios individuais e coletivos advindos desse fortalecimento ${ }^{23}$. Neste sentido, alguns autores têm mostrado que pertencer a comunidades com capital social mais alto está associado com níveis mais elevados de $\mathrm{AF}$, inclusive de lazer ${ }^{24-25}$.

O estudo colombiano mostrou que outras estruturas que não são específicas de prática de AF no lazer também são importantes, como por exemplo, o acesso ao transporte público coletivo ${ }^{22}$. Este fato ressalta a importância de parte do escore ter sido composto pelos itens de segurança para o deslocamento, pois as pessoas precisam se deslocar e se apoderar dos locais próximos às residências nos bairros de moradia para praticarem AF. Itens como faixas de pedestres e semáforos são importantes para a segurança no trânsito, principalmente pelo modo violento e desrespeitoso como os motoristas dirigem os seus veículos nas grandes cidades ${ }^{26}$ e pelos problemas causados por este modo de dirigir, como um elevado número de mortes por atropelamentos em regiões periféricas de São Paulo ${ }^{27}$. No 
ano de 2011, a Prefeitura de São Paulo lançou o Programa de Proteção ao Pedestre <http://www.preferenciaavida.com.br $>$ que tem como objetivo diminuir o número de acidentes por atropelamentos por meio da valorização das faixas de pedestres. Foram implantadas e revitalizadas 14 mil faixas de pedestres e 833 orientadores de travessia e 2,4 mil agentes de trânsito estão contribuindo com o programa.

Calçadas adequadas também são muito importantes para o deslocamento e estão associadas com a prática de AF em países como o Brasil ${ }^{11}$. Atualmente no município de São Paulo há uma lei (15.442/2011) que foi regulamentada recentemente e torna obrigatória a construção e a manutenção de calçadas adequadas para o deslocamento, oferecendo instruções por meio de uma cartilha padronizada. Espera-se que esta lei contribua para uma melhora nas calçadas da cidade de São Paulo. Outro item importante é a iluminação pública, que está entre as maiores queixas de pessoas que residem em áreas de baixo nível socioeconômico no município de São Pau$1 \mathrm{l}^{28}$. O indicador de iluminação (quantidade de luminárias) também está relacionado com a percepção de segurança. Evidências obtidas em parte desta amostra de adultos nesta mesma região mostrou que percepção de segurança adequada está relacionada com a prática de caminhada como forma de deslocamento?.

A poluição ambiental entrou no indicador como uma variável negativa (quantidade de ruas com esgoto a céu aberto). Regiões periféricas de São Paulo ainda têm uma menor cobertura de rede geral de tratamento de esgoto sanitário em comparação com as regiões mais centrais. Menores níveis de poluição ambiental percebida estão associados com a prática de AF em adultos de Ermelino Matarazzo ${ }^{20}$.

Os estudos de países de renda média (Curitiba, no Brasil, e Bogotá, na Colômbia) optaram por analisar a associação das variáveis ambientais com a prática de AF separadamente sem criar um escore de ambiente ${ }^{12,22}$. Os estudos de países de renda alta têm usado escores compostos, principalmente por um indicador denominado de walkability ${ }^{29}$, em que são utilizados dados como a densidade residencial (quantidade de residências por área), conectividade das ruas (intersecções) e uso misto do solo (quantidade de estabelecimentos comerciais, de serviços, indústrias, locais para lazer e razões entre áreas comerciais e de serviços por área). O presente estudo foi realizado numa área de baixo nível socioeconômico dentro do município de São Paulo e optou-se por se criar um escore conjunto com as variáveis mais significativas, pois acredita-se que somente um conjunto de mudanças ambientais poderão influenciar de forma mais significativa o nível de AF no lazer e chegar mais próximo do contexto de ambientes amigáveis para a prática de $\mathrm{AF}^{1,19}$.

Algumas limitações devem ser ressaltadas. Os dados do presente estudo foram derivados de inquérito de base domiciliar. As residências foram selecionadas com objetivo de entrevistas domiciliares e o foco não era avaliar o ambiente de forma objetiva para a prática de AF. Além disso, esta proposta de escore de ambiente construído necessita ser testada e avaliada 
no contexto de análise multinível, separando as variáveis individuais das variáveis contextuais. Uma última limitação refere-se a aplicação do escore de ambiente construído em outras regiões. Estes resultados são muito específicos para o local onde as variáveis foram avaliadas e só devem ser comparados com regiões muito similares a Ermelino Matarazzo.

Para finalizar, conclui-se que para um estudo inicial de caráter exploratório, este escore de ambiente construído para a prática de AF no tempo de lazer é uma contribuição que pode servir para gestores e pesquisadores discutirem propostas de indicadores e atributos relacionados a ambientes saudáveis em regiões similares a Ermelino Matarazzo na zona leste de São Paulo.

\section{Agradecimentos}

Agradecemos a Fundação de Amparo à Pesquisa do Estado de São Paulo (FAPESP) e ao Conselho Nacional de Pesquisas (CNPq) pelos auxílios concedidos para a realização deste estudo.

\section{REFERÊNCIAS BIBLIOGRÁFICAS}

1. Sallis JF, Cervero RB, Ascher W, Henderson KA, Kraft MK, Kerr J. An ecological approach to creating active living communities. Annu Rev Public Health 2006;27:297-322.

2. Brownson RC, Hoehner CM, Day K, Forsyth A, Sallis JF. Measuring the built environment for physical activity: state of the science. Am J Prev Med 2009;36(4 Suppl): S99-123.

3. Sallis JF. Measuring physical activity environments: a brief history. Am J Prev Med 2009;36(4 Suppl):S86-92.

4. Hino AAF, Reis RS, Florindo AA. Built environment and physical activity: a brief review of evaluation methods. Rev Bras Cineantropom Des Hum 2010;12(5): 387-94.

5. Ingram GK, Carroll A. The spatial structure of Latin American cities. J Urban Econ 1981; 9(2):257-73.

6. Bauman AE, Reis RS, Sallis JF, Wells JC, Loos RJ, Martin BW. Correlates of physical activity: why are some people physically active and others not? Lancet;380(9838):258-71.

7. Parra DC, Hoehner CM, Hallal PC, Ribeiro IC, Reis R, Brownson RC, et al. Perceived environmental correlates of physical activity for leisure and transportation in Curitiba, Brazil. Prev Med 2011;52(3-4):234-8.

8. Gomes GA, Reis RS, Parra DC, Ribeiro I, Hino AA, Hallal PC, et al. Walking for leisure among adults from three Brazilian cities and its association with perceived environment attributes and personal factors. Int J Behav Nutr Phys Act 2011;8(1):111.

9. Florindo AA, Salvador EP, Reis RS, Guimaraes VV. Percepção do ambiente e prática de atividade física em adultos residentes em região de baixo nível socioeconômico. Rev Saude Publica 2011;45(2):302-10.

10. Salvador EP, Reis RS, Florindo AA. Practice of walking and its association with perceived environment among elderly Brazilians living in a region of low socioeconomic level. Int J Behav Nutr Phys Act 2010;7:67.

11. Hallal PC, Reis RS, Parra DC, Hoehner C, Brownson RC, Simoes EJ. Association between perceived environmental attributes and physical activity among adults in recife, Brazil. J Phys Act Health 2010;7(2 Suppl):S213-22.

12. Hino AA, Reis RS, Sarmiento OL, Parra DC, Brownson RC. The built environment and recreational physical activity among adults in Curitiba, Brazil. Prev Med 2011;52(6):419-22. 
13. Salvador EP, Florindo AA, Reis RS, Costa EF. Perception of the environment and leisure-time physical activity in the elderly. Rev Saude Publica 2009;43(6):972-80.

14. Hallal PC, Victora CG, Wells JC, Lima RC. Physical inactivity: prevalence and associated variables in Brazilian adults. Med Sci Sports Exerc 2003;35(11):1894-900.

15. Organización Mundial de la Salud. Recomendaciones mundiales sobre actividad física para la salud. Genebra: Organización Mundial de la Salud; 2010.

16. Florindo AA, Guimaraes VV, Cesar CL, Barros MB, Alves MC, Goldbaum M. Epidemiology of leisure, transportation, occupational, and household physical activity: prevalence and associated factors. J Phys Act Health 2009;6(5):625-32.

17. Bortoni WL, Florindo AA, Salvador EP, Reis RS. Desenvolvimento e reprodutibilidade de um instrumento de avaliação objetiva do ambiente para aplicação em estudos de atividades físicas. Rev Bras Atividade Fisica Saude 2009;14(1):38-47.

18. Pikora T, Giles-Corti B, Bull F, Jamrozik K, Donovan R. Developing a framework for assessment of the environmental determinants of walking and cycling. Soc Sci Med 2003;56(8):1693-703.

19. Brennan Ramirez LK, Hoehner CM, Brownson RC, Cook R, Orleans CT, Hollander $\mathrm{M}$, et al. Indicators of activity-friendly communities: an evidence-based consensus process. Am J Prev Med 2006;31(6):515-24.

20. Florindo AA, Salvador EP, Reis RS. Physical activity and its relationship with perceived environment among adults living in a region of low socioeconomic level. J Phys Act Health 2013: no prelo.

21. Hocking RR. The analysis and selection of variables in linear regression. Biometrics 1976;32(1):1-49.

22. Gomez LF, Sarmiento OL, Parra DC, Schmid TL, Pratt M, Jacoby E et al. Characteristics of the built environment associated with leisure-time physical activity among adults in Bogota, Colombia: a multilevel study. J Phys Act Health 2010;7 (2 Suppl):S196-203.

23. Milani C. Teorias do capital social e desenvolvimento local: lições a partir de experiências de Pintadas (Bahia, Brasil) IV Conferência Regional ISTR-LAC; San Jose - Costa Rica 2003. p. 1-30.

24. Legh-Jones H, Moore S. Network social capital, social participation, and physical inactivity in an urban adult population. Soc Sci Med 2012;74:1362-7.

25. Lindström, M. Social capital, desire to increase physical activity and leisure time physical activity: a population-based study. Public Health 2011;25(7):442-7.

26. Vanderbilt T. Por que dirigimos assim? Rio de Janeiro: Elsevier; 2009.

27. Secretaria Municipal de Saúde de São Paulo. Programa de Aprimoramento das Informações de Mortalidade no Município de São Paulo: indicador de mortes por atropelamentos de 2009. Disponível em: < http://ww2.prefeitura.sp.gov.br/cgi/ deftohtm.exe?secretarias/saude/TABNET/SIM/obito.def > [30 jul 2012].

28. Folha de São Paulo. DNA Paulistano. Publifolha: São Paulo; 2008.

29. Frank LD, Sallis JF, Saelens BE, Leary L, Cain K, Conway TL, et al. The development of a walkability index: application to the Neighborhood Quality of Life Study. Br J Sports Med 2010;44(13):924-33.

Endereço para correspondência Alex Antonio Florindo Rua Arlindo Bettio, 1000. Ermelino Matarazzo.

CEP: 03828-000 - São Paulo, SP. Brasil Email: aflorind@usp.br 